\title{
A new tool for ancient artefact conservation studies: Electron Diffraction Tomography to study blue corrosion product in Chinese Bronze sample
}

\author{
P.P. Das ${ }^{1}$, E. Mugnaioli², Q. Wang ${ }^{3}$, S. Nicolopoulos ${ }^{1}$, M. Gemmi ${ }^{2}$ \\ ${ }^{1}$ NanoMEGAS SPRL, Rue Émile Claus 49 bte 9, 1050, Brussels, Belgium, \\ ${ }^{2}$ Center for Nanotechnology Innovation@NEST, Istituto Italiano di Tecnologia, Piazza San Silvestro 12, 56127, Pisa, Italy, \\ ${ }^{3}$ Institute of Cultural Heritage, Shandong University, 72 Jimo Binhailu, Qingdao 266237, China
}

partha@nanomegas.com

The in-depth understanding of properties, manufacturing process and conservation of archaeological artefacts very often requires a reliable structural characterization. Non-destructive techniques, like X-ray diffraction and different kinds of spectroscopies (Raman, IR etc.), are usually employed to the study such materials. In recent years, the scientific community has also shown a renewed interest in characterization methods based on Transmission Electron Microscope (TEM); like High Resolution Transmission Electron Microscopy (HRTEM), Electron Diffraction (ED), Energy Dispersive X-ray Spectroscopy (EDX) and Electron Energy Loss Spectroscopy (EELS); which provide structural and chemical information at $\mathrm{nm}$ scale using very small quantities of material. In particular, we have shown how emerging diffraction techniques like Electron Diffraction Tomography (EDT) and Phase \& Orientation mapping in TEM can be applied for the study of nanocrystalline phases present in Greek amphorisks, Roman glass tesserae and several Maya pigments [1,2].

We are now working on the structural characterisation of corrosion products from different archaeological artefacts using TEM. In most cases, due to the very small quantity of existing corrosion products, conventional diffraction methods (single crystal X-ray diffraction or powder X-ray diffraction) are not suitable for a proper structural characterization. In particular, the pale blue corrosion products that form on ancient copper alloy artefacts have been subject of research for past several years, though the exact nature of such corrosion products have not yet been determined. Here, we present an innovative study based on EDT of a blue corrosion product forming on the surface of a Chinese Bronze artefact of the Shang dynasty (British Museum Collections).

For this study, a thin electron beam-transparent lamella ( 8 x 10 micron) was prepared from a larger poly-crystalline sample $(100 \times 200$ micron) using Focused Ion Beam (FIB). Subsequently, EDX data was collected by a Scanning Electron Microscope (SEM). A very high quantity of $\mathrm{Cu}$ was observed, together with other elements like $\mathrm{Ca}, \mathrm{P}$ and $\mathrm{O}$. The thin lamella was then used for EDT study using TEM. EDT data was collected by stepwise rotation of the crystal around an arbitrary axis coupled with beam precession [Fig. 1]. From the EDT data, a monoclinic unit cell $\left(a=23.0 \AA, b=5.4 \AA, c=10.3 \AA, \beta=94.1^{\circ}\right.$, space group $\left.P 2 / c\right)$ was determined. Interestingly the obtained unit cell does not match with any of the blue corrosion products reported in literature. Using the extracted intensity from EDT data, a preliminary structure was determined, which closely resembles one of the blue minerals, nissonite.

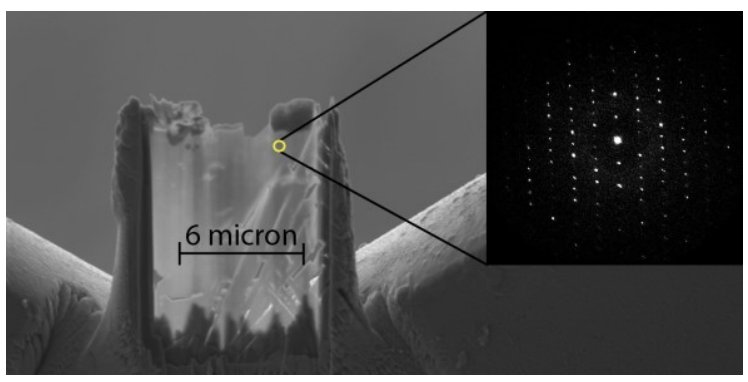

Figure 1: Electron diffraction pattern from the thin lamella sample during EDT data collection.

We believe that novel structural characterisations using EDT in future may not only help in the understanding of corrosion processes in ancient artefacts, but also can contribute to their optimum conservation and eventually provide information about their provenance.

[1] Zacharias, N., Karavassili, F., Das, P. P., Nicolopoulos, S., Oikonomou, A., Galanis, A., Rauch, E., Arenal, R., Portillo, J., Roque, J., Casablanca, J. \& Margiolaki, I. (2018) Microchemical Journal, 138, 19.

[2] Nicolopoulos, S., Das, P. P., Pérez, A. G., Zacharias, N., Cuapa, S. T., Alatorre, J. A. A., Mugnaioli, E., Gemmi, M. \& Rauch, E. F. (2019) Scanning.

Keywords: Transmission Electron Microscopy, EDT, Ancient artefact, Corrosion, Conservation 